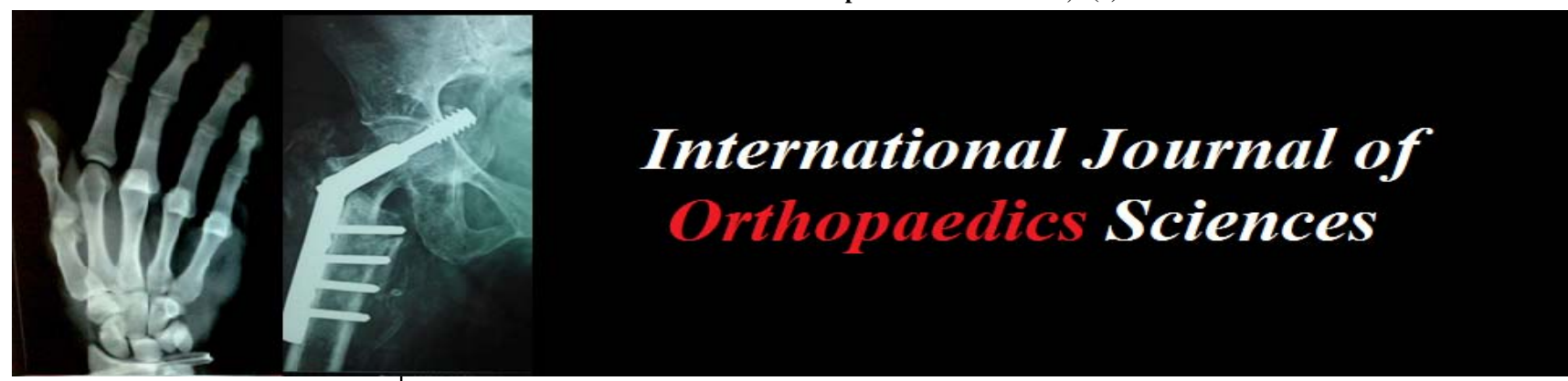

ISSN: $2395-1958$

IJOS 2017; 3(1): 162-164

(C) 2017 IJOS

www.orthopaper.com

Received: 26-11-2016

Accepted: 27-12-2016

SK Rai

Classified Specialist,

Department of Orthopedics

INHS Asvini, Colaba Mumbai,

India

Major Rohit Vikas

Graded Specialist, Department

of Orthopedics INHS Asvini,

Colaba Mumbai, India

\section{Rohit Varma}

Graded Specialist, Department of

Orthopedics INHS Asvini,

Colaba Mumbai, India

\section{Dr. Naveen Shijale}

Assistant Professor, Department of Orthopaedics, INHS Asvini,

Colaba Mumbai, India

\section{Application of PHILOS humerus plate for a distal Tibial fracture: An effective alternative}

\author{
SK Rai, Major Rohit Vikas, Rohit Varma and Dr. Naveen Shijale
}

DOI: http://dx.doi.org/10.22271/ortho.2017.v3.i1c.26

Abstract

Ankle fractures are among the most common fracture injury in young and old and, if not managed correctly, can be debilitating in the long term. The most common ankle fracture is an isolated malleolar fracture that represents $66 \%$ of all ankle fractures. Bi-malleolar fractures account for $25 \%$ with the remainder being tri-malleolar ${ }^{[1]}$.

Management of these fractures varies depending on the severity, stability and pattern of fracture. Options can range from conservative management by plaster to external fixation; especially in the case of an open fracture. Unfortunately, the ideal fixation option (most commonly used plate) implant is not always available in operation theatre. The PHILOS plate can be second best alternative and effective option for fixation of these fractures.

Keywords: PHILOS, osteosynthesis plate, fracture distal tibia, malleolar fracture

\section{Introduction}

Ankle fractures are among the most common fracture injury and, if not managed correctly, can be debilitating in the long term. The most common ankle fracture is an isolated malleolar fracture that represents $66 \%$ of all ankle fractures. Bi-malleolar fractures account for $25 \%$ with the remainder being tri-malleolar ${ }^{[1]}$.

Management of these varies depending on the severity, stability and pattern of fracture. Options can range from conservative management (plaster of Paris) to external fixation; especially in the case of an open fracture. Unfortunately, the ideal fixation option (most commonly used plate) implant is not always available in operation theatre. The PHILOS plate can be second best alternative and effective option for fixation of these fractures.

\section{Patients and Methods}

In order to determine the efficacy of PHILOS plate as alternative implants for distal tibia fracture we included 20 patients in our studies between Jan 2014 to Jan 2016 with minimum 01 year follow up.

We included only recent fractures and closed fracture which was distal most extra articular fracture of Tibia, where nail cannot be placed and when there was no idea tibial plate available. (AO type - extra articular-43 A1, 43 A2, and 43 A3 type fractures)

All 13 patients had similar type of injury i.e twisting of ankle and fall while walking, running or playing. In 05 patients who were old female sustained injury due to fall in bathroom at home. 02 patients sustained injury due to fall from staircase. All patients had a lateral malleolar fracture with a vertical shear of the medial malleolus and comminution at the joint line. The fracture may be classified as a Weber A, or Lauge Hansen type 2 (Fig. 1) or AO 43 A1, 2, and 3 .

These fracture were unstable and required fixation. In 02 patients there were excessive soft tissue with bleb formation, the operation was delayed for 6 days (Fig. 2B). All operation was performed under a Spinal Anaesthesia with the patient supine and a sandbag placed under the buttock. A tourniquet was applied in all cases and a lateral approach to the fibula was taken with standard washout and fracture reduction achieved. The distal fibular fracture was fixed using LCDCP.
Lt Col SK Rai

Graded Specialist, Department of Orthopedics INHS Asvini,

Colaba Mumbai, India 


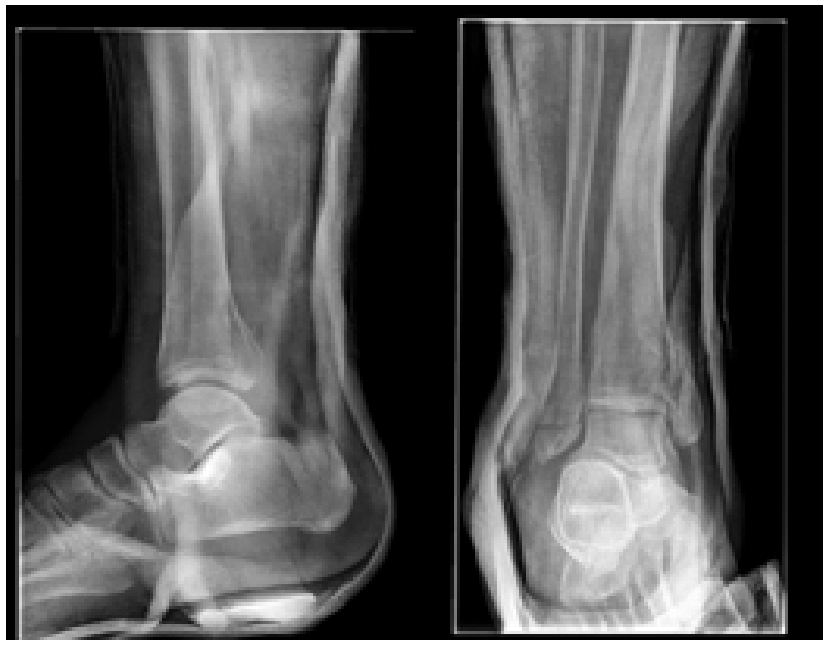

Fig 1: AP and lateral radiographs of the fracture post-reduction and immobilization.

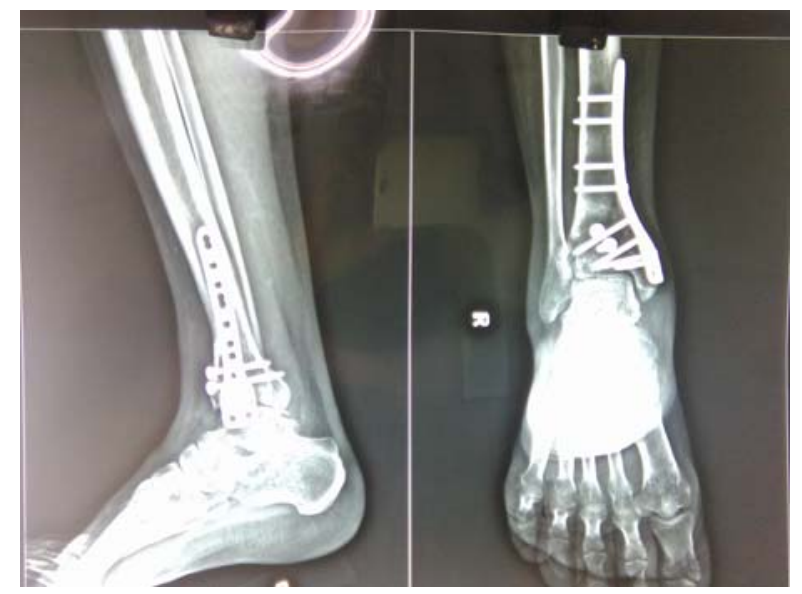

Fig 2A: AP and lateral radiographs of the fracture post-fixation.

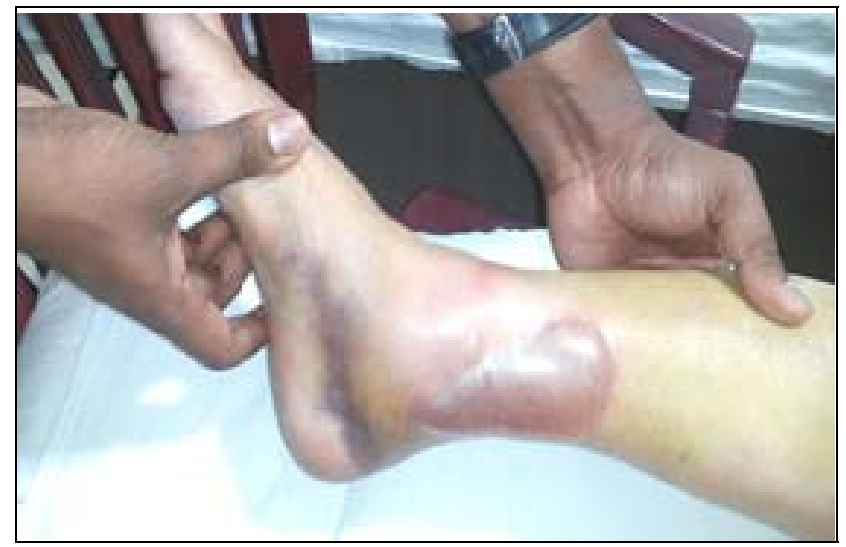

Fig 2B: bleb formation

The distal tibia was approached via a direct medial approach. This revealed an extensively comminuted fracture extending proximally. The fracture site was identified, reduced and held in place with $2 \times 2.0 \mathrm{~mm} \mathrm{k}$-wires. These medial fracture was not amenable to screw fixation or naling. The next best alternative was to use a PHILOS plate as it appeared to fit the contours appropriately. An intraoperative decision was made to use a $3.5 \mathrm{~mm} \mathrm{LCP}{ }^{\circledR}$ Proximal Humerus Plate (PHILOS) plate. The plate fit the distal tibial contours well once the fracture was reduced. Three proximal non-locking cortical screws and multiple distal locking screws were applied producing a stable construct (Fig. 2).

The wounds were then washed out with normal saline and closed with 2-0 Vicryl to deep tissue, and interrupted 3-0 ethilon to skin. Gelonet and non-adhesive dressings were covered with crepe and a backslab.

Post-operatively, antibiotics were prescribed as per our hospital protocol (two doses of Teicoplanine 400mg) and $48 \mathrm{~h}$ of strict elevation. All patients was instructed to remain nonweight bearing for 6 weeks.

There were no complications in any patient. All patients' wounds were inspected at $2^{\text {nd }}, 9^{\text {th }}$ and on finally on $14^{\text {th }}$ days post-operative, and were discharged after 15 day after suture removal. All patients were given below knee POP for 6 weeks. The next review at 6 weeks following surgery. At this review, the cast was removed and ankle ROM was started and was allowed to walk with crutched non-weight bearing with ankle physiotherapy.

All patients were call to review after 6 weeks again and then after 3 months and then after 6 months (12 months postsurgery).

\section{Results}

There were no complications in any patient. All patients regained ankle ROM fully by 12 weeks

All patients were call to review after 6 weeks again and then after 3 months and then after 6 months (12 months postsurgery).
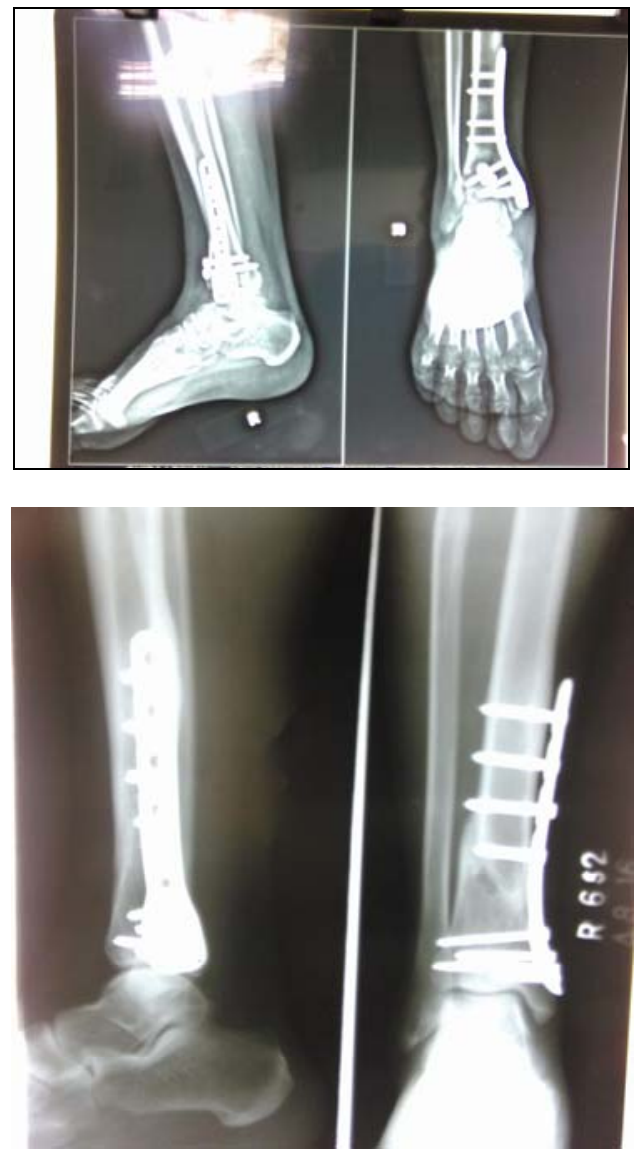

Fig 3: Fracture has fully united (01 year follow up)

No patients has developed any post-operative infection or wound dehiscence. All patients were followed up minimally upto 12 moths and all were shown fracture union, 


\section{Discussion}

In our review of the literature, the only use of the PHILOS plate in the ankle was for ankle arthrodesis ${ }^{[2]}$, other uses were limited to the fracture proximal Humerus only ${ }^{[4]}$.

Locking plate technology, advances in prostheses and fixation methods have changed the way we look at fractures. The principle of holding a fracture in place with locking screws and plates means that the distance of the plate from the bone is less important, in contrast to the importance of adequate proximal and distal hold ${ }^{[3]}$. The stable monoblock is achieved by threading of the screws into the plate and the bone. Although the locking plate does not need to be opposed to the bone with these prostheses, problems may occur if it is not close enough. Any prostheses too big or distant from the ankle will result in difficult closures, tenting skin and wound-healing problems.

The other difficulty faced by the trauma surgeon is the financial implications of implants and stock management. It is becoming more prevalent for stock the implant to be ordered 'just-in-time' as a means of reducing stock costs. This can easily result in the wrong implants being ordered, or the appropriate implants not being available.

From our series, it can be seen that in situations where the appropriate implants is not available, an alternatives need to be considered. A PHILOS plate is an acceptable and near best substitute for distal tibial fracture fixation in cases where other implants like suitable nail and tibial plates is unavailable, or nailing cannot be done because of fracture is comminuted and is too close to joint line. Based on our series and promising results, we can now recommend that PHILOS can be used when right or ideal implants for distal tibia is not available.

\section{References}

1. Koval KJ, Zuckerman JD. Handbook of Fractures. 3rd edn. London: Lippincott Williams \& Wilkins, 2006.

2. Ahmad J, Pour AE, Raikin SM. The modified use of a proximal humeral locking plate for tibiotalocalcaneal arthrodesis. Foot Ankle Int. 2007; 28:977-83.

3. Smith WR, Ziran BH, Anglen JO, Stahel PF. Locking Plates: Tips \& Tricks. J Bone Joint Surg Am. 2007; 89A:2298-307.

4. Martinez AA, Cuenca J, Herrera A. Philos plate fixation for proximal humeral fractures J Orthop Surg (Hong Kong). 2009; 17(1):10-4. 